\title{
Bronchiectasis Rheumatoid Overlap Syndrome Is an Independent Risk Factor for Mortality in Patients With Bronchiectasis
} A Multicenter Cohort Study

Anthony De Soyza, MD, PhD; Melissa J. McDonnell, MD; Pieter C. Goeminne, MD, PhD; Stefano Aliberti, MD, PhD; Sara Lonni, MD; John Davison, RN; Lieven J. Dupont, MD, PhD; Thomas C. Fardon, MD; Robert M. Rutherford, MD; Adam T. Hill, MD; and James D. Chalmers, MD, PhD

BACKGROUND: This study assessed if bronchiectasis (BR) and rheumatoid arthritis (RA), when manifesting as an overlap syndrome (BROS), were associated with worse outcomes than other BR etiologies applying the Bronchiectasis Severity Index (BSI).

METHODS: Data were collected from the BSI databases of 1,716 adult patients with BR across six centers: Edinburgh, United Kingdom (608 patients); Dundee, United Kingdom ( $\mathrm{n}=286$ ); Leuven, Belgium $(\mathrm{n}=253)$; Monza, Italy $(\mathrm{n}=201)$; Galway, Ireland $(\mathrm{n}=242)$; and Newcastle, United Kingdom $(n=126)$. Patients were categorized as having BROS (those with RA and BR without interstitial lung disease), idiopathic BR, bronchiectasis-COPD overlap syndrome (BCOS), and "other" BR etiologies. Mortality rates, hospitalization, and exacerbation frequency were recorded.

RESULTS: A total of 147 patients with BROS ( $8.5 \%$ of the cohort) were identified. There was a statistically significant relationship between BROS and mortality, although this relationship was not associated with higher rates of BR exacerbations or BR-related hospitalizations. The mortality rate over a mean of 48 months was $9.3 \%$ for idiopathic BR, $8.6 \%$ in patients with other causes of BR, $18 \%$ for RA, and $28.5 \%$ for BCOS. Mortality was statistically higher in patients with BROS and BCOS compared with those with all other etiologies. The BSI scores were statistically but not clinically significantly higher in those with BROS compared with those with idiopathic BR (BSI mean, 7.7 vs 7.1, respectively; $P<.05$ ). Patients with BCOS had significantly higher BSI scores (mean, 10.4), Pseudomonas aeruginosa colonization rates (24\%), and previous hospitalization rates (58\%).

CONCLUSIONS: Both the BROS and BCOS groups have an excess of mortality. The mechanisms for this finding may be complex, but these data emphasize that these subgroups require additional study to understand this excess mortality.

CHEST 2017; 151(6):1247-1254

KEY WORDS: bronchiectasis; COPD; mortality; rheumatoid arthritis

ABBREVIATIONS: BCOS $=$ bronchiectasis and COPD overlap syndrome; $\mathrm{BR}=$ bronchiectasis; $\mathrm{BROS}=$ bronchiectasis-rheumatoid arthritis overlap syndrome; BSI = Bronchiectasis Severity Index; RA = rheumatoid arthritis

AFFILIATIONS: From the Adult Bronchiectasis Service \& Sir William Leech Centre for Lung Research (Dr De Soyza and $\mathrm{Mr}$ Davison), Freeman Hospital, Heaton, Newcastle, UK; Institute of Cellular Medicine (Drs De Soyza and McDonnell), Newcastle
University, Newcastle, UK; Department of Respiratory Medicine (Drs McDonnell and Rutherford), Galway University Hospitals, Galway, Ireland; University Hospital Gasthuisberg (Drs Goeminne and Dupont), Respiratory Medicine, Leuven, Belgium; Department of Pathophysiology and Transplantation (Dr Aliberti), University of Milan Fondazione IRCCS Ca' Granda Ospedale Maggiore Policlinico, Milan, Italy; Department of Health Science (Dr Lonni), University of Milan Bicocca, 
Non-cystic fibrosis bronchiectasis (hereafter referred to as bronchiectasis [BR]) is a chronic respiratory disorder characterized by recurrent cough, sputum production, and respiratory infections. ${ }^{1}$ Pathologically, patients have abnormally dilated bronchi leading to impairment of host defenses, chronic infection with bacteria, and airways inflammation. ${ }^{2,3}$

Rheumatoid arthritis (RA) is a common autoimmune disease associated with many extra-articular features. RA has numerous pulmonary complications, including interstitial lung diseases, which may lead to "traction" BR; the association between RA and BR without interstitial lung disease (hereafter referred to as BROS) is well recognized. Studies note a significantly higher prevalence of symptomatic BR in patients with RA (approximately $3 \%$ ) compared with $0.03 \%$ in the general population. ${ }^{4}$ Supporting this finding are high-resolution CT scanning studies consistently reporting a high prevalence (up to $30 \%$ ) of radiologic evidence of BR in RA populations. ${ }^{5,6}$

Earlier single-center studies have suggested that patients with BROS may have a worse clinical course than those patients with BR due to other etiologies. We identified that when compared with patients with RA alone, patients with BROS have higher indices of RA activity (eg, 28-item Disease Activity Scores), demonstrating worse RA and higher levels of RA seropositivity. ${ }^{7}$

The goal of the present study, therefore, was to determine if BROS was associated with poorer outcomes compared with BR without RA. Defining the clinical severity of BR had been problematic until scoring indices such as the Bronchiectasis Severity Index (BSI) became available. ${ }^{8}$ We sought to assess the mortality, frequency of exacerbations, hospital admissions, reported health-related quality of life, and BSI scores in an international cohort comparing BROS vs BR without RA. Idiopathic BR was used as a benchmark because of its prevalence and a perception that this etiologic group may have better outcomes. ${ }^{1}$ Because BR and COPD overlap syndrome (BCOS) has been linked to excess mortality, this second group was used as an additional reference group. ${ }^{9}$

\section{Patients and Methods}

\section{Multicenter Assessment of BR Severity}

Six independent cohorts of patients were collected from specialist BR services in Edinburgh, Dundee, and Newcastle (United Kingdom), Leuven (Belgium), Monza (Italy), and Galway (Ireland); the average follow-up was 4 years. ${ }^{8,10}$ Consecutive adult patients were enrolled on the basis of a diagnosis of BR made by using high-resolution CT

Clinica Pneumologica, Monza, Italy; Tayside Respiratory Research Group (Drs Fardon and Chalmers), University of Dundee, Dundee, UK; and the Department of Respiratory Medicine (Dr Hill), Royal Infirmary of Edinburgh and the University of Edinburgh, Edinburgh, UK.

FUNDING/SUPPORT: This study was funded in part by the Medical Research Council (MRC). Dr De Soyza acknowledges a Higher Education Funding Council for England senior lectureship, support from the National Institute for Health Research Biomedical Research Centre, and MRC funding [MR/L0011263/1] for a UK multicenter registry (BRONCH-UK). Dr Chalmers acknowledges fellowship support from the MRC and the Wellcome Trust. Dr McDonnell acknowledges fellowship support from the European Respiratory Society (ERS)/European Lung Foundation and the Health Research Board, Ireland. Dr Dupont is a senior research fellow of the FWO. The following acknowledge support from an ERS Clinical Research Collaboration in bronchiectasis (European Multicentre Bronchiectasis Audit and Research Collaboration): Drs De Soyza, Chalmers, Aliberti, Goeminne, and McDonnell.

CORRESPONDENCE TO: Anthony De Soyza, MD, PhD, Adult Bronchiectasis Service \& Sir William Leech Centre for Lung Research, Freeman Hospital, Heaton, Newcastle, NE7 7DN, UK; e-mail: anthony.de-soyza@ncl.ac.uk

Copyright (C 2017 American College of Chest Physicians. Published by Elsevier Inc. All rights reserved.

DOI: http://dx.doi.org/10.1016/j.chest.2016.12.024 scans and a clinical history consistent with BR. ${ }^{1}$ Patients were excluded if they had active malignancy at enrollment, cystic fibrosis, active mycobacterial disease (including active nontuberculous mycobacteria), HIV, or a primary diagnosis of pulmonary fibrosis/ sarcoidosis with secondary traction BR. Patients with BCOS were not included within the Edinburgh cohort because of their cohort building protocol. Patient cohort recruitment was approved at each individual center; by the South East Scotland Research Ethics Committee, Research ethics service multicenter ethics, IRAS 12324 and by NRES, UK 12/NE/0298, CA 128 Clinical research committee, Galway. ${ }^{8,10}$

\section{Etiologic Categorization}

The underlying etiology of BR was determined following testing recommended by the British Thoracic Society guidelines. ${ }^{1}$ This testing includes serologic and clinical assessments for RA.

BROS required a diagnosis of both $\mathrm{BR}$, as noted earlier, and RA, defined according to the 2010 American College of Rheumatology and the European League Against Rheumatism RA criteria ${ }^{11}$ and local prevailing clinical guidelines. Patients were grouped into the BROS category irrespective of which of the two conditions preceded the other.

Patients were pragmatically categorized as having BCOS based on evidence of airflow obstruction and smoking $>20$ pack-years. The presence of emphysema on CT scanning was not a prerequisite.

Postinfectious causes were attributed when a clear history of BR following an acute infectious episode was reported. ${ }^{1}$ Inflammatory bowel disease and allergic bronchopulmonary aspergillosis-associated etiologic categories were applied when a clear history and/or appropriate serologic and history were reported, respectively. Idiopathic was attributed as a diagnostic grouping in the absence of any recognized etiology. "Other" BR was a grouping of categories 
that included all remaining etiologic groups (eg, immunodeficiencyassociated BR, including those taking immunoglobulin replacement, those with ciliary dyskinesia).

\section{Clinical Assessments}

At the time of clinical assessment, all patients were clinically stable with no antibiotic use in the preceding 4 weeks. All patients underwent spirometry ( $\mathrm{FEV}_{1}$ and $\left.\mathrm{FVC}\right)$ according to European Respiratory Society guidelines, with the highest of three technically satisfactory measurements recorded.

\section{Radiologic Severity}

Radiologic severity of BR was assessed by using a modified Reiff score, which has been used previously in BR studies. ${ }^{8,12,13}$ The score assesses the number of lobes involved (with the lingula considered to be a separate lobe) and the degree of bronchial dilatation (tubular, 1; varicose, 2; and cystic, 3) with a maximum score of 18 and minimum score of 1 . There was no minimum Reiff score for patients to be entered into the cohorts.

\section{Bacteriology}

As previously described, all bacteriologic studies were performed by using local culture protocols on spontaneous early morning sputum samples. ${ }^{3}$ The definition of chronic persistent infection was the isolation of potentially pathogenic bacteria in sputum culture on $\geq 2$ occasions, at least 3 months apart in a 1 -year period. ${ }^{13-15}$ The microorganism grown most frequently over the study period was classified as the predominant pathogen. The clinical standards were sputum sampling at six monthly or more frequent intervals at clinic reviews.

\section{BSI Scores}

As previously described, BSI scores were grouped as follows: scores of 0 to 4 represent mild BR; scores of 5 to 8 indicate moderate BR; and scores $>8$ represent severe $\mathrm{BR}^{8}$

\section{End Points}

At the end of the follow-up periods, mortality was determined through notes review and examining national death records. Survival status was confirmed for $100 \%$ of participants, although exact date of death was not available for all deceased patients.

Exacerbations were defined according to the British Thoracic Society description as an acute deterioration with worsening and/or systemic upset. $^{1}$ Severe exacerbations were defined as those needing hospitalization. The frequency of exacerbations requiring antibiotic treatment was determined from clinic records and patient histories, and this information was verified against primary care prescription records.

\section{Statistical Analysis}

Normally distributed data are presented as mean \pm SD, and nonnormally distributed data are presented as medians with interquartile ranges. The $\chi^{2}$ test and Mann-Whitney $U$ test were used for comparison of categorical and numerical data, respectively. For comparisons of $>2$ groups, one-way ANOVA or the Kruskal-Wallis test was used as appropriate. For all analyses, a $P$ value $<.05$ was considered statistically significant. Independent relationships between BROS and BCOS with mortality were assessed by using multivariable logistic regression, adjusting for the BSI. Data are presented as ORs with 95\% CIs. Kaplan-Meier survival curves and Cox proportional hazards regression were performed for survival.

The discrimination of the BSI for predicting mortality in BROS was assessed by using the area under the receiver-operating characteristic curve. Sensitivity analyses were performed to determine if outcomes were different across all three BSI categories (mild, moderate, and severe). In addition, we applied calibration analysis, an analysis to determine whether scoring systems perform similarly in a different population compared with the baseline population. As a sensitivity analysis to determine the validity of pooling cohorts, random effects meta-analysis was used. Data were pooled by using the Mantel-Haenszel method, and heterogeneity was assessed by using Higgins $I^{2}$ test and Cochran's Q test.

\section{Results}

\section{Multicenter Assessment}

Data were collected from 1,716 adult patients with BR across six centers in Western Europe. The data are displayed in Figure 1 and Table 1. The patients' median age was 65 years, with a female predominance, and the most common etiologic groups were idiopathic and postinfectious, suggesting these were broadly representative of BR cohorts previously reported. ${ }^{1}$

Overall, BROS was present in $8.5 \%$ of the cohort, whereas BCOS was present in $12 \%$ of the cohorts that included BCOS during cohort building. The mean exacerbation frequency was $>2$ exacerbations per year, and all cohorts reported a history of hospitalization in at least $20 \%$ of patients. Chronic Pseudomonas aeruginosa infection was present in a mean of $13 \%$ of patients overall. The mean BSI scores for each center ranged from 6 to 9. Previous data have

shown that this finding is consistent with patients with moderate to severe BR. ${ }^{8}$ The center with the highest hospital admission rate (Newcastle) also had the highest observed mean BSI score (ie, 9.6).

\section{Comparison Between BROS and Non-RA Patients With Bronchiectasis}

The comparisons between BROS and other groups are shown in Table 2. In general, patients with BROS were similar in terms of age and sex distribution except compared with the BCOS group, who were significantly older and significantly more likely to be male. The BSI scores were statistically significantly higher in the BROS group compared with the idiopathic group and other BR groups, although all remained within the moderate severity category of the BSI (scores, 5-8). Radiologic burden of disease was not significantly different across all groupings, with three lobes involved as an average. Notably, both the BCOS group and the BROS group had 


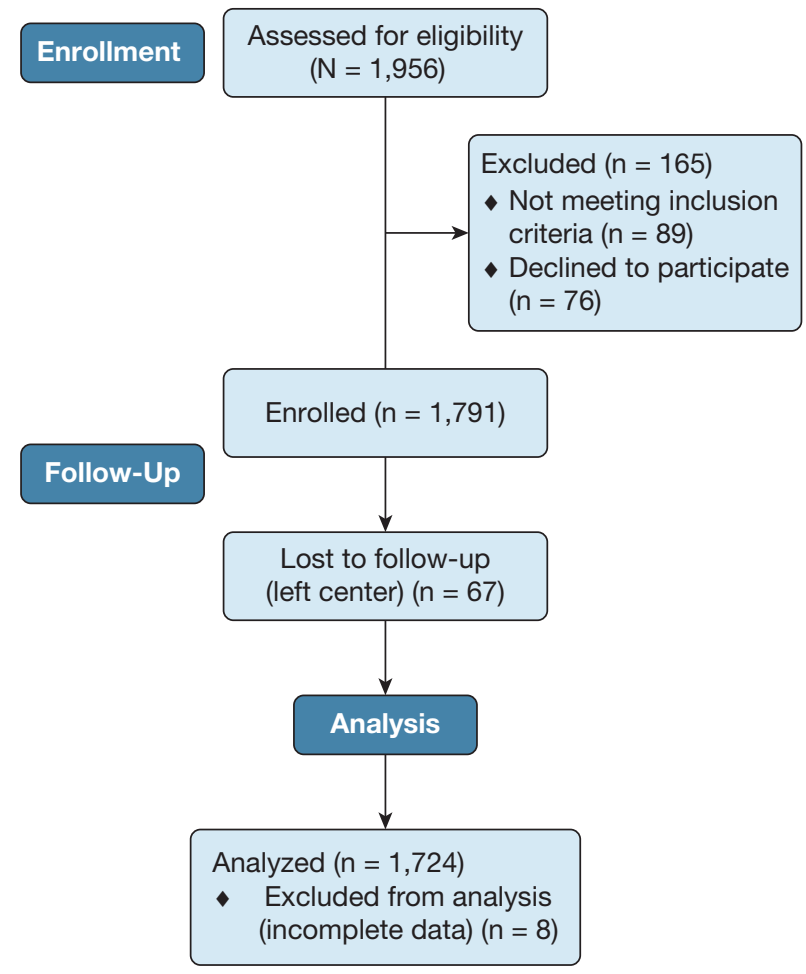

Figure 1 - CONSORT 2010 flow diagram.

statistically significantly more exacerbations and previous BR-related hospitalizations than the idiopathic BR group (BROS mean number of exacerbations 2.4 and $26.1 \%$ hospitalization rate, $\mathrm{BCOS} 2.7$ exacerbations and $58.4 \%$ vs BR 1.8 and $25.1 \%, P<.05$; Table 2 ). As expected, the mean $\mathrm{FEV}_{1} \%$ predicted was both statistically and clinically significantly lower in the BCOS group, in part reflecting the need for airflow obstruction to be present in this diagnostic grouping.

\section{Outcomes in BROS}

The mortality rate over a mean of 48 months of followup was $8.6 \%$ in patients with other causes of BR, $9.3 \%$ in idiopathic BR, $18 \%$ for RA, and $28.5 \%$ for BCOS. There was no significant difference in follow-up duration among any of the four cohorts to explain the differences in mortality (mean, 46, 48, 47, and 47 months, respectively) (Fig 2).

Using logistic regression, there was a significant univariate association between RA and increased mortality (OR, 1.82 [95\% CI, 1.15-2.89]; $P=.01$ ). This scenario persisted after multivariable adjustment for BSI (OR, 1.83 [95\% CI, 1.11-3.02]; $P=.01$ ). The relationship was greater in the fully adjusted model (including etiology, all BSI individual components) (OR, 2.03 [95\% CI, 1.19-3.44]; $P=.009$ ). COPD was also independently associated with worse outcome in all models adjusted (OR, 2.47 [95\% CI, 1.55-3.92] in the fully adjusted model). No other etiologies were independently associated with outcome (HosmerLemeshow goodness-of-fit test, $P=.7$ [indicating excellent model fit]).

There was, however, no significant relationship between RA and hospital admission risk during follow-up (OR, 0.84 [95\% CI, 0.42-1.67]; $P=.6$ ). There was no significant relationship between RA and more frequent exacerbations according to multiple linear regression (adjusted for BSI: estimate, 0.15; SE, 0.18; $P=.5$ ).

The results were confirmed by using Cox proportional hazards regression. The hazard ratio for RA and mortality was 1.88 (95\% CI, 1.11-3.21; $P=.01$ ). The Kaplan-Meier survival curve is shown both for BCOS and BROS in Figure 2.

\section{Prediction}

Despite clear variations in mortality rates associated with different etiologies, the BSI showed good discrimination in patients with BROS. It yielded an area under the receiver-operating characteristic curve of 0.77 (95\% CI, 0.67-0.87; $P<.0001$ ).

We also applied a calibration analysis to determine whether the BSI scoring systems perform similarly well in a different population, such as BROS compared with the overall BR population. RA was associated with an increased mortality risk across all BSI subgroups: OR of 2.57 (95\% CI, 0.48-13.9) in low-risk patients, 2.1

(95\% CI, 0.8-5.5) in intermediate-risk patients, and 1.64 (95\% CI, 0.83-3.3) in high-risk patients (interaction test, $P=.8)$. This analysis indicated that RA increases the risk across the full spectrum of BR severity categories and should be considered additive to the BSI.

\section{Validation of the Pooled Analysis}

Using random effects meta-analysis of the six cohorts, RA was associated with increased mortality (OR, 1.70 [95\% CI, 1.07-2.70]; $P=.02$ ). Importantly, there was no heterogeneity in this relationship across all six studies $\left(I^{2}=0 \%\right.$; Cochran Q test, $\left.P=.6\right)$.

\section{Discussion}

$\mathrm{BR}$ and RA are undoubtedly linked and may present in patients in a variety of temporal and causal ways. ${ }^{4,5,7} \mathrm{BR}$ seems to predispose to later RA, and "BRRA" could be used to define this syndrome. ${ }^{5}$ Patients with RA are known to develop BR as their articular disease progresses and could be described as "RABR." A third group could 
TABLE 1 ] Details of the European BR Cohorts

\begin{tabular}{|c|c|c|c|c|c|c|}
\hline Variable & $\begin{array}{l}\text { Leuven } \\
\text { (Belgium) }\end{array}$ & $\begin{array}{l}\text { Galway } \\
\text { (Ireland) }\end{array}$ & $\begin{array}{l}\text { Monza } \\
\text { (Italy) }\end{array}$ & $\begin{array}{l}\text { Edinburgh } \\
\text { (UK) }\end{array}$ & $\begin{array}{l}\text { Newcastle } \\
\text { (UK) }\end{array}$ & $\begin{array}{l}\text { Dundee } \\
\text { (UK) }\end{array}$ \\
\hline Total & $253(100)$ & $242(100)$ & $201(100)$ & $608(100)$ & $126(100)$ & $286(100)$ \\
\hline \multicolumn{7}{|l|}{ Demographic } \\
\hline Age, median (IQR), y & $68(56-78)$ & $63(53-71)$ & $68(59-73)$ & $67(58-75)$ & $61(54-69)$ & $68(61-75)$ \\
\hline Male sex & $127(50)$ & $76(31)$ & 80 (39) & $243(40)$ & $51(41)$ & $115(42)$ \\
\hline \multicolumn{7}{|l|}{ Etiology ${ }^{a}$} \\
\hline Idiopathic & $78(31)$ & $98(40)$ & 79 (39) & $261(42)$ & $52(41)$ & $124(43)$ \\
\hline Postinfective & 50 (19) & $41(17)$ & $51(25)$ & $207(34)$ & $28(22)$ & $51(17)$ \\
\hline ABPA & $15(6)$ & $5(2)$ & $4(2)$ & $49(8)$ & $8(6)$ & $31(11)$ \\
\hline $\mathrm{BCOS}$ & $42(17)$ & $26(11)$ & $49(24)$ & $0^{\mathrm{b}}$ & $15(12)$ & $7(2)$ \\
\hline Immunodeficiency & $18(7)$ & $13(5)$ & $9(4)$ & $6(1)$ & $14(11)$ & $16(6)$ \\
\hline BROS & $25(10)$ & $55(23)$ & $2(1)$ & $44(7)$ & $11(9)$ & $10(4)$ \\
\hline IBD & $5(2)$ & $4(2)$ & $6(3)$ & $14(2)$ & $2(1)$ & $8(3)$ \\
\hline \multicolumn{7}{|l|}{ Severity markers } \\
\hline Exacerbations/y & $1.8 \pm 2.0$ & $3.2 \pm 1.3$ & $1.9 \pm 1.9$ & $1.7 \pm 2.0$ & $3.4 \pm 1.7$ & $2.1 \pm 1.8$ \\
\hline $\begin{array}{l}\text { Previous hospital } \\
\text { admissions }\end{array}$ & $67(26)$ & $63(26)$ & $56(27)$ & $133(21)$ & $74(58)$ & $66(23)$ \\
\hline $\begin{array}{l}\text { \% Pseudomonas } \\
\text { aeruginosa }\end{array}$ & $20(8)$ & $35(14)$ & 39 (19) & $70(12)$ & $13(10)$ & $37(14)$ \\
\hline $\begin{array}{l}\text { Lobes involved on } \\
\text { CT scanning }\end{array}$ & $2.9 \pm 1.3$ & $2.7 \pm 1.3$ & $2.8 \pm 1.4$ & $3.0 \pm 1.6$ & $2.8 \pm 1.4$ & $3.2 \pm 1.6$ \\
\hline Mean $\mathrm{FEV}_{1} \%$ predicted & $70.1 \pm 27$ & $77.5 \pm 24$ & $71.7 \pm 35$ & $72.6 \pm 25$ & $64.0 \pm 27$ & $72.1 \pm 26$ \\
\hline Mean BSI score & $6.7 \pm 4.8$ & $7.2 \pm 4.4$ & $7.2 \pm 4.5$ & $7.3 \pm 4.8$ & $9.6 \pm 4.9$ & $7.1 \pm 4.5$ \\
\hline
\end{tabular}

Data are presented as No. (\%) or mean \pm SD unless otherwise indicated. ABPA = allergic bronchopulmonary aspergillosis; BCOS = bronchiectasis and COPD overlap syndrome; BR = bronchiectasis; BROS = bronchiectasis-rheumatoid arthritis overlap syndrome; BSI = bronchiectasis severity index; IBD = inflammatory bowel disease; IQR = interquartile range.

aLess frequent etiologies are not shown.

bPatients with BCOS were excluded from this cohort.

include those who coincidentally have both conditions without any causal relationship. Reflecting concerns over recall bias and inaccuracy in pinpointing the onset of a particular condition (in contrast to the time when it was diagnosed), we have opted to use the terminology BROS to encompass all three of these scenarios.
To our knowledge, this study is the first multicenter international study to apply the recently validated BSI to define the severity of $\mathrm{BR}$ in patients with comorbid RA. We report data in almost 150 patients with BROS from a 1,716-patient cohort followed up over an average of 4 years with BR in the largest and only multicenter study to

TABLE 2 ] Comparison Between BROS and Non-RA Patients With BR

\begin{tabular}{l|c|c|c|c}
\hline Variable & BROS & Idiopathic BR & BCOS & Other BR \\
\hline Age, median (IQR), y & $69(60-76)^{\mathrm{a}}$ & $67(58-74)^{\mathrm{a}}$ & $73(65-78)^{\mathrm{b}}$ & $64(55-72)^{\mathrm{a}, \mathrm{b}}$ \\
Sex & $34.3 \% \mathrm{male}^{\mathrm{a}}$ & $38.2 \% \mathrm{male}^{\mathrm{a}}$ & $70.0 \% \mathrm{male}^{\mathrm{b}}$ & $38.4 \% \mathrm{male}$ \\
\hline Exacerbations/y & $2.4 \pm 1.9$ & $1.8 \pm 1.9^{\mathrm{a}, \mathrm{b}}$ & $2.7 \pm 2.0$ & $2.2 \pm 2.0$ \\
\hline Previous hospital admissions & $26.1 \%^{\mathrm{a}}$ & $25.1 \%^{\mathrm{a}}$ & $58.4 \%^{\mathrm{b}}$ & $23.7 \%^{\mathrm{a}}$ \\
\hline Pseudomonas aeruginosa infection & $14.3 \%^{\mathrm{a}}$ & $14.7 \%^{\mathrm{a}}$ & $24.1 \%^{\mathrm{b}}$ & $14 \%^{\mathrm{a}}$ \\
Lobes involved on CT scanning & $3.0 \pm 1.5$ & $2.8 \pm 1.5$ & $3.1 \pm 1.4$ & $3.0 \pm \pm 1.5$ \\
Mean FEV $\%$ predicted & $76 \% \pm 25^{\mathrm{a}}$ & $76 \% \pm 25^{\mathrm{a}}$ & $51 \% \pm 22^{\mathrm{b}}$ & $74 \% \pm 25^{\mathrm{a}}$ \\
\hline Mean BSI score & $7.7 \pm 4.6^{\mathrm{a}}$ & $7.1 \pm 4.6^{\mathrm{a}, \mathrm{b}}$ & $10.4 \pm 4.5^{\mathrm{b}}$ & $6.9 \pm 4.3^{\mathrm{a}, \mathrm{b}}$ \\
\hline \hline
\end{tabular}

Data are presented as mean \pm SD unless otherwise indicated. See Table 1 legend for expansion of abbreviations.

${ }^{a} P<.05$ compared with BCOS.

${ }^{\mathrm{b}} \mathrm{P}<.05$ compared with BROS. 


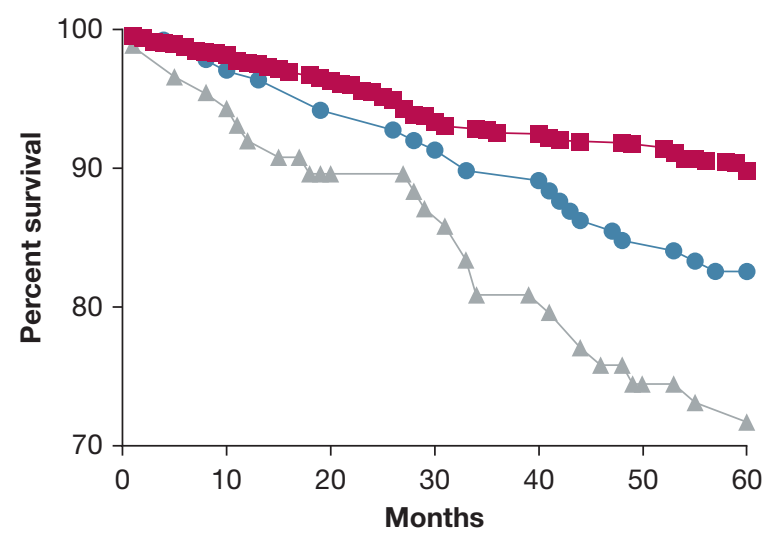

$\rightarrow-\mathrm{BR}-\mathrm{BROS} \rightarrow \mathrm{BCOS}$

Figure 2 - Kaplan-Meier survival curve is shown both for BCOS and BROS. $B C O S=$ bronchiectasis and COPD overlap syndrome; $B R=$ bronchiectasis; $B R O S=$ bronchiectasis-rheumatoid arthritis overlap syndrome.

date to define the impact of RA in BR. We benchmarked this group against a group increasingly recognized as having poorer outcomes, namely those with BCOS, and those often perceived to have more favorable outcomes, namely "idiopathic" BR. We found, however, that although there was a statistically significantly higher BSI score in the BROS group compared with the idiopathic BR group (BSI mean, 7.7 vs. 7.1; $P<.05$ ), this outcome was not likely to be clinically significant because the mean BSI scores were both within the moderate BSI category (BSI score, 5-8).

Importantly however, the present study found that BROS was significantly associated with increased mortality compared with idiopathic BR syndrome. Indeed, mortality with BROS approached that seen in BCOS. ${ }^{9,16}$ Using multiple modeling methods, we showed that the mortality risk over 4 years was increased by approximately $80 \%$ and, when adjusted for all components of the BSI, that the OR reached 2.0 , indicating a doubling of mortality risk. This effect was replicated in survival analyses, confirming that BROS is associated with higher mortality.

Importantly, this outcome seems independent of the rates of hospitalization, nonhospitalized exacerbations, and spirometric and radiologic markers of disease burden.

The co-existence of BR and RA has previously been suggested to have major clinical significance. In 1997, a single-center UK study reported that patients with both BR and RA (BROS) had greatly elevated standardized mortality ratios 7.3 times higher than the general population, 5 times that of patients with RA alone, and 2.4 times that of patients with BR over 5 years. ${ }^{17}$ Our observed mortality rates herein were $18 \%$, and the OR for mortality was slightly less than that reported in the aforementioned study. Careful review of this previous research suggests potential case ascertainment bias with a more severe BROS subgroup selected: only 32 patients with BROS were identified from their RA cohort of 3,000 (1\%). Their reported prevalence rate was lower than we observed (approximately 8\%) and contrasts to more recent studies suggesting prevalence rates ranging from $3 \%$ to $30 \%$ radiologically. Nevertheless, a single-center casecontrol study of patients recruited from 1999 to 2002 reported an excess of mortality over an 11-year follow-up period. ${ }^{18}$ The patients with BROS also had a poorer prognosis in terms of survival after diagnosis of RA (hazard ratio, 8.6 [95\% CI, 1.5-48.2]; $P=.014$ ) and from birth (hazard ratio, 9.6 [95\% CI, 1.1-81.7]; $P=.039$ ).

Divergence in mortality rates was seen within the first 5 years in this study. Collectively, these previous data and our international multicenter observations support BROS as a risk for poorer outcomes.

The reasons for this finding may be distinct to the pulmonary disease component as suggested by the similar rates of exacerbation and lung function seen between BROS and idiopathic BR noted in the present study. This effect may be more clearly observed in those with milder BR as suggested by our sensitivity analysis. It is possible that the treatments used for RA, which include powerful immunosuppressant drugs, may affect survival, but our study was not designed to define the reasons for poorer outcomes. In this study, we did not have funding to collect detailed information on the management of RA and were therefore unable to assess the role of this factor in the observed increased mortality. Notably, however, in our previous research, we found no significantly different rates of diseasemodifying antirheumatic drug therapy between patients with RA and BROS in an intensively characterized UK cohort. ${ }^{19}$ We did, however, report greater rates of autoantibody seropositivity, inflammatory markers, and joint involvement, suggesting the BROS syndrome is associated with greater immune activation and systemic inflammation. ${ }^{7,19}$ This finding is noteworthy because RA has been associated with an excess of cardiovascular deaths and is now incorporated as an independent risk factor in the cardiovascular QRISK2 scoring system. ${ }^{20}$ $\mathrm{BR}$ has also been recently linked with excessive cardiovascular risk, ${ }^{21}$ and this link may be an underpinning mechanism for excess mortality in BROS, with additive cardiovascular risk driven by each proinflammatory comorbidity. This theory requires 
further mechanistic research that was not possible in the present study because only limited data collection was possible.

We also showed that the BSI scoring system still predicts poorer mortality outcomes in those with BROS and that the effects are seen across the range of BSI categories. RA is certainly an additive and independent predictor of severity/death, and etiology may need incorporation in future risk stratification systems. To benchmark the outcomes in BROS, a previously described BR etiology associated with poor outcomes was used.

We showed that BCOS has an elevated mortality risk ( $28 \%$ risk of death over 4 years), which is much higher than that reported in the selected population recruited into the Towards a Revolution in COPD Health (TORCH) study (patients who had an average $\mathrm{FEV}_{1}$ of approximately $60 \%$ [ $15 \%$ mortality over 3 years]). ${ }^{22}$ The mortality rates in the $\mathrm{BCOS}$ population were high and in the order of those reported in patients with Global Initiative for Chronic Obstructive Lung Disease stage II/ III COPD (or those within body mass index, airflow obstruction, dyspnea, exercise capacity [BODE] index quartile 3) in the BODE index cohort and in other studies. ${ }^{23,24}$ We extend the findings of Gatheral et $\mathrm{al}^{25}$ showing that BCOS is associated with a high hospital admission rate (58\% in this series) and that persistent $P$ aeruginosa infection is common in BCOS

(24\% herein). In contrast to an article from the United Kingdom, which did not report an excess of mortality in BCOS compared with COPD alone, ${ }^{25}$ we confirm research from other investigators ${ }^{9,16,26}$ that BCOS is associated with excess mortality compared with other BR etiologies. These differences may be explained by the comparator groups; Gatheral et al ${ }^{25}$ compared patients with BCOS vs patients with relatively severe COPD, whereas in the other studies and our present study, the comparator group has been $\mathrm{BR}$, often including those with mild disease. ${ }^{26}$ Our definition of BCOS may have incorrectly categorized patients as having idiopathic BR who previously smoked as BCOS. Nevertheless, our pragmatic definition seems to have confirmed the findings reported from single centers. ${ }^{9,16,26}$ Consensus exists regarding the need to better define BR phenotypes and predictors of mortality. ${ }^{27,28}$

One area to focus on is BCOS, a syndrome that is clearly adversely prognostic yet difficult to define precisely, where the mechanisms leading to adverse outcomes are imprecise (as reviewed by Hurst et $\mathrm{al}^{28}$ ). BROS clearly is another area also requiring better understanding. We did not have prescription records of immunosuppressive therapies to target RA in this patient population; such therapies may influence both infection rates and possibly mortality in the setting of BROS. These data will be prospectively collected in UK national and European observational cohorts and should allow future associations to be explored (www.bronch.ac.uk). ${ }^{29}$

Our study has inherent limitations in addition to those relating to concomitant medications. We excluded patients with active nontuberculous mycobacterial disease and patients with known RA-related interstitial lung disease. These factors may have contributed to the differences in the BSI scores between groups. We cannot, however, exclude the possibility of "missed" cases of $B R O S$ being incorrectly classified as idiopathic BR in any of the cohorts, although serologic testing for RA was conducted in all cohorts. The pooling of data from multiple centers may be regarded as a limitation because there was some heterogeneity in the populations, such as the exclusion of patients with BCOS from the Edinburgh cohort (this choice reflected an a priori decision at that recruiting center). ${ }^{8}$ Nevertheless, in our sensitivity analysis, no significant heterogeneity was demonstrated in the relationship between BROS and mortality, and we therefore regard the robustness of this finding across multiple centers as a strength and not as a weakness. We did not assess RA serology repeatedly, only doing so at a patient's first clinic review or when new symptoms prompted a clinical suspicion of RA. It is therefore possible that these patients with BR may have inadvertently included some patients with subclinical or early-stage RA who should have been placed in the BROS category. Lastly, our mortality data did not compare outcomes in BROS vs a cohort of patients with RA alone; the recorded cause of death was also not included. These data will be highly relevant to future studies.

\section{Conclusions}

In the largest cohort studied to date, both BROS and BCOS were shown to be associated with poorer outcomes, and they should be investigated further as a priority in longitudinal and mechanistic studies to assess drivers of mortality. ${ }^{28,29}$ The current data support the premise that patients with BROS are at higher risk of premature death, and a multidisciplinary approach involving chest and rheumatology physicians is needed. Patients with BROS with "mild" BR defined radiologically by extent or by using composite scoring systems may need closer monitoring than those with other etiologies causing BR. 


\section{Acknowledgments}

Author contributions: A. D. S. is the guarantor of the paper. A. D. S., J. C., S. A., P. G., and M. J. M. designed the study; M. J. M., A. D. S., and J. C. drafted the manuscript; and A. D. S., M. J. M., and J. C. conducted the statistical analyses. The coauthors collected the primary data and revised the drafts.

Financial/nonfinancial disclosures: The authors have reported to CHEST the following: A. D. S. has received speaking fees and served on Industry advisory panels for Bayer Healthcare, AstraZeneca, GlaxoSmithKline and Pfizer. He has received grants from Gilead, Novartis, GlaxoSmithKline, Forrest labs and Chiesi. S. A. has received speaking fees and served on industry advisory committees for Bayer Healthcare, AstraZeneca, Griffols, Aradigm Corporation, Basilea, Zambon, Novartis Raptor, Chiesi, and Actavis UK Ltd. J. D. C. has received grant funding from AstraZeneca, GlaxoSmithKline, and Pfizer and fees for consulting or speaking from AstraZeneca, Pfizer, Napp, and Boehringer-Ingelheim. None declared (M. J. M., P. C. G., S. L., J. D., L. J. D., T. C. F., R. M. R., A. T. H.).

Role of sponsors: The sponsor had no role in the design of the study, the collection and analysis of the data, or the preparation of the manuscript.

Other contributions: The authors acknowledge Alberto Pesci, MD, from the Health Science Department, University of Milan Bicocca, and Paul McAlinden, Freeman Hospital, for assistance with data collection.

\section{References}

1. Pasteur MC, Bilton D, Hill AT. British Thoracic Society guideline for non-CF bronchiectasis. Thorax. 2010;65(suppl 1) i1-i58.

2. Chalmers JD, Hill AT. Mechanisms of immune dysfunction and bacterial persistence in non-cystic fibrosis bronchiectasis. Mol Immunol. 2013;55(1): 27-34.

3. Chalmers JD, Smith MP, McHugh B, Doherty C, Govan JRW, Hill AT. Short and long term antibiotic therapy reduces airway and systemic inflammation in nonCF bronchiectasis. Am J Respir Crit Care Med. 2012;186(7):657-665.

4. Wilczynska MM, Condliffe AM, McKeon DJ. Coexistence of bronchiectasis and rheumatoid arthritis: revisited. Respir Care. 2013;58(4):694-701

5. Wilsher M, Voight L, Milne D, et al. Prevalence of airway and parenchymal abnormalities in newly diagnosed rheumatoid arthritis. Respir Med. 2012;106(10):1441-1446.
6. Mohd Noor N, Mohd Shahrir MS, Shahid MS, Abdul Manap R, Shahizon Azura AM, Azhar Shah S. Clinical and high resolution computed tomography characteristics of patients with rheumatoid arthritis lung disease. Int Rheum Dis. 2009;12(2):136-144.

7. Perry E, Stenton C, Kelly C, Eggleton P, Hutchinson D, De Soyza A. RA autoantibodies as predictors of rheumatoid arthritis in non-cystic fibrosis bronchiectasis patients. Eur Respir J. 2014;44:1082-1085.

8. Chalmers JD, Goeminne P, Aliberti S, et al. The bronchiectasis severity index. An international derivation and validation study. Am J Respir Crit Care Med. 2014;189(5):576-585.

9. Martínez-García MA, de la Rosa Carrillo D, Soler-Cataluña JJ, et al Prognostic value of bronchiectasis in patients with moderate-to-severe chronic obstructive pulmonary disease. Am J Respir Crit Care Med. 2013;187(8): 823-831.

10. McDonnell MJ, Aliberti S, Goeminne PC, et al. Multidimensional severity assessment in bronchiectasis: an analysis of seven European cohorts. Thorax. 2016;71(12):1110-1118.

11. Aletaha D, Neogi T, Silman AJ, et al. 2010 Rheumatoid arthritis classification criteria: an American College of Rheumatology/ European League Against Rheumatism collaborative initiative. Arthritis Rheum. 2010;62:2569-2581.

12. Reiff DB, Wells AU, Carr DH, Cole PJ, Hansell DM. CT findings in

bronchiectasis: limited value in distinguishing between idiopathic and specific types. AJR Am J Roentgenol. 1995; 165(2):261-267.

13. Pasteur MC, Helliwell SM, Houghton SJ, et al. An investigation into causative factors in patients with bronchiectasis. Am J Respir Crit Care Med. 2000;162(4 pt 1): 1277-1284.

14. Chalmers JD, McHugh BJ, Doherty C, et al. Mannose-binding lectin deficiency and disease severity in non-cystic fibrosis bronchiectasis: a prospective study. Lancet Respir Med. 2013;1(3):224-232.

15. Lee TW, Brownlee KG, Conway SP, Denton M, Littlewood JM. Evaluation of a new definition for chronic Pseudomonas aeruginosa infection in cystic fibrosis patients. J Cyst Fibros. 2003;2:29-34.

16. Goeminne PC, Scheers H, Decraene A, et al. Risk factors for morbidity and death in non-cystic fibrosis bronchiectasis: a cross-sectional analysis of CT diagnosed bronchiectatic patients. Respir Res. 2012;13:21.

17. Swinson DR, Symmons D, Suresh U, Jones M, Booth J. Decreased survival in patients with co-existent rheumatoid arthritis and bronchiectasis. $\mathrm{Br} J$ Rheumatol. 1997;36(6):689-691.

18. Puéchal X, Génin E, Bienvenu T, Le Jeunne C, Dusser DJ. Poor survival in rheumatoid arthritis associated with bronchiectasis: a family-based cohort study. PLoS One. 2014;9(10):e110066.

19. Perry E, Eggleton P, De Soyza A, Hutchinson D, Kelly C. Increased disease activity, severity and autoantibody positivity in rheumatoid arthritis patients with co-existent bronchiectasis [published online ahead of print July 22, 2015]. Int J Rheum Dis. http://dx.doi.org/10.1111 /1756-185X.12702.

20. Hippisley-Cox J, Coupland C, Robson J, Brindle P. Derivation, validation, and evaluation of a new QRISK model to estimate lifetime risk of cardiovascular disease: cohort study using QResearch database. BMJ. 2010;341:c6624.

21. Navaratnam V, Millett E, Hurst JR, et al. Bronchiectasis and the risk of cardiovascular disease: a population-based study. Thorax. 2017;72(2):161-166.

22. Calverley PM, Anderson JA, Celli B, et al; TORCH Investigators. Salmeterol and fluticasone propionate and survival in chronic obstructive pulmonary disease. N Engl J Med. 2007;356(8): 775-789.

23. Celli BR, Cote CG, Marin JM, et al. The body-mass index, airflow obstruction, dyspnea, and exercise capacity index in chronic obstructive pulmonary disease. $N$ Engl J Med. 2004:350(10):1005-1012.

24. Ko FW, Tam W, Tung AH, et al A longitudinal study of serial BODE indices in predicting mortality and readmissions for COPD. Respir Med. 2011;105(2):266-273.

25. Gatheral T, Kumar N, Sansom B, et al. COPD-related bronchiectasis; independent impact on disease course and outcomes. COPD. 2014;11(6):605-614.

26. Goeminne PC, Nawrot TS, Ruttens D, Seys S, Dupont LJ. Mortality in non-cystic fibrosis bronchiectasis: a prospective cohort analysis. Respir Med. 2014;108(2): 287-296.

27. De Soyza A, Brown JS, Loebinger MR. Research priorities in bronchiectasis. Thorax. 2013;68(7):695-696.

28. Hurst JR, Elborn JS, De Soyza A; BRONCH-UK Consortium. COPDbronchiectasis overlap syndrome. Eur Respir J. 2015;45(2):310-313.

29. Chalmers JD, Aliberti S, Polverino E, et al The EMBARC European Bronchiectasis Registry: protocol for an international observational study. ERJ Open Research. 2016;2(1). 